\title{
PACR: Position-Aware Protocol for Connectivity Restoration in Mobile Sensor Networks
}

\author{
Rab Nawaz Jadoon $\mathbb{D}^{1,2}$ Adnan Anwar Awan, ${ }^{3}$ Muhammad Amir Khan, ${ }^{3}$ WuYang Zhou (D), \\ and Aqdas Naveed Malik ${ }^{4}$ \\ ${ }^{1}$ Key Laboratory of Wireless-Optical Communication, University of Science and Technology China, Hefei 230027, China \\ ${ }^{2}$ Department of Computer Science, COMSATS University Islamabad, Abbottabad Campus 22060, Pakistan \\ ${ }^{3}$ Department of Electrical and Computer Engineering, COMSATS University Islamabad, Abbottabad Campus 22060, Pakistan \\ ${ }^{4}$ Department of Electronic Engineering, ISRA University I-10/4 Islamabad, 46000, Pakistan
}

Correspondence should be addressed to Rab Nawaz Jadoon; rabnawaz@mail.ustc.edu.cn and WuYang Zhou; wyzhou@ustc.edu.cn

Received 30 September 2020; Revised 24 November 2020; Accepted 15 December 2020; Published 31 December 2020

Academic Editor: Claudia Campolo

Copyright (c) 2020 Rab Nawaz Jadoon et al. This is an open access article distributed under the Creative Commons Attribution License, which permits unrestricted use, distribution, and reproduction in any medium, provided the original work is properly cited.

\begin{abstract}
Wireless Sensor Networks (WSNs) have gained global attention in recent times due to their vast applications in various fields. These networks can face the disruption of data transmission due to sensor node failures when placed in harsh, inaccessible, and adverse environments such as battlefields or monitoring in enemy territory. The specific tasks performed by the collaboration among the sensor nodes in WSNs by internode connectivity may be terminated. Besides this, due to the failure of sensor nodes, the area covered by the network may be limited, which can cause damage to the objectives for such a network, as there might be an unaware danger in the lost area. Connectivity is a big problem in mobile WSNs due to the mobility of nodes. Researchers have developed a lot of algorithms that are capable enough for connectivity problems, but they do not emphasize the loss of coverage. We try to fill these gaps by proposing the new hybrid algorithm PACR (Position-Aware protocol for Connectivity Restoration). The concept behind PACR is the same as a person who writes his will before death on a deathbed. In the same way, when the sensor energy is below the threshold, it is converted into a recovery coordinator and generates a recovery plan. This accelerates the recovery by decreasing the time needed for failure identification. For the recovery process, the neighbor's nodes do not travel to the exact position of the failed node. Instead, they just move to the distance where they can build communication links with other nodes. This greatly prolongs the network lifetime. The simulation results show that PACR outperforms other techniques present in the literature.
\end{abstract}

\section{Introduction}

Wireless Sensor Networks (WSNs) are an important topic of research these days. WSNs have densely distributed sensor nodes with low cost, having low power. These nodes send their sensed data to sink nodes located in WSNs $[1,2]$. Data like temperature, pressure, light, soil composition, humidity, detection of chemical and biological attacks, sound recording, etc. is sensed by these nodes. The sensed data is collected, organized, and analyzed to perform different necessary actions. The motivation behind the development of Wireless Sensor Networks is to perform necessary actions in battlefield surveillance, monitoring industrial processes, controlling machine health, health care, security, surveillance, etc. [3, 4]. Sensor nodes consist of sensors, a battery, microcontroller, and transceiver for communication of data. These nodes may be mounted on robotic vehicles, and such nodes are known as mobile nodes. The main advantage of WSNs is to sense data in a harsh environment. These harsh environments include battlefields, volcanic mountains, and any other environment where human intervention is impossible. Sensor nodes can be deployed randomly or preplanned in the area of interest (AOI). In some situations, node deployment is preplanned, i.e., they do not need to configure themselves for positioning in the network. On the other hand, in harsh environments, sensor nodes are deployed randomly so that 
they can configure themselves into a communication network and perform their task. So, various techniques have been proposed for these two kinds of deployments in the literature so far. Details of such techniques are given in the literature review section. The coverage area and connectivity of sensor nodes play a very important role in any network design to perform specific tasks. Coverage area is important because after deployment for performing their task, nodes have to sense data around their surroundings in a specific area. That specific area is known as the coverage area. Connectivity is the ability of the sensor nodes to find out the routes to transmit the sensed data to the sink node. If the connectivity is lost due to any reason, then the sensed node cannot reach the sink node and data processing cannot be done. So, the important task for which the network is deployed cannot be done properly; hence, keeping connectivity is important.

Sensor nodes may fail due to battery depletion, malfunction, or destruction in harsh environments. These failures may lead to serious damage to WSNs. A network will lose the coverage area and connectivity in case of failure. Hence, the main objectives of WSNs will be compromised. The topology of the network will also change due to the failure of nodes. Therefore, we need to detect the failure and should adopt some method to restore connectivity. Batteries can be changed and nodes can be replaced for maintaining the connectivity and for lifetime improvement, but in harsh environments, it is difficult or almost impossible to change the batteries of sensor nodes or to replace failed nodes. When a node fails, we have to adopt some methods to recover it. Sometimes, a large number of nodes fail at a time which affects the task being performed by the network and reduces the significance of that network. So, this issue drew the researcher's attention towards the solution of simultaneous failed node recovery. So far, different strategies have been proposed in the literature for the retrieval of node failures. In the first approach, the topology of the network may be reorganized by the repositioning of sensor nodes to restore the connectivity. Such techniques supported the self-healing of WSNs. These techniques used a distributive manner, whereas the second approach uses multiple-relay sensor nodes to reinstate the multiple split segments. In the third technique, by mobile mules, data is collected and is used to determine the cut vertexes and transmission of that data from one segment of the network to another in WSNs [5].

A roaming location secrecy approach for users of position-based services (PBSs) using machine learning techniques is suggested in [6]. The writers propose a triphase method for wandering PBS users. By combining decision trees and $k$-nearest neighbor, it identifies user location and estimates user destination using hidden Markov models along with the sequence of position tracks. Also, a mobile edge computing service approach that will ensure the timely implementation of PBSs is adopted by the proposed model. The benefits of a mobile edge service strategy provide location anonymity and low latency in the proximity of roaming users through networking and processing facilities.

An energy-conscious green adversary model is proposed in [7] for its use in smart industrial environments by preserving secrecy. While it is possible to jointly upgrade the hard- ware and software elements of cyber-physical networks to reduce their energy consumption, this focuses on issues of place conservation and material secrecy. The authors provide several testimonials to help cyber-physical defence experts and analysts based on literature results (assumptions, adversary priorities, and capabilities). To minimize the expense of communication and computing with each query, the proposed model running on real-time, anticipatory positionbased query scheduling facilitates the minimization of energy usage. Furthermore, we calculate the move/approval slots that are needed for each query to prevent degrading slots. Experimental results show that the new approach would minimize energy consumption by up to five times by existing practices.

The main purpose explained in [8] is to increase the network lifetime by extending the operating sensor lifespan as well as transferring the data obtained from the super node to the sink. In this article, the Bat Algorithm (BA) is used to select the best node for the monitoring sensor and the effective path to reducing energy consumption. Simulation results and comparison with other algorithms indicate the supremacy of the suggested algorithm. The simulation results of the proposed algorithm reveal that the algorithm proposed was able to decrease the power consumption of the network and improve the service life of the network. The proposed algorithm could also, on average, outperform the comparable algorithms by $27 \%$.

The whale optimization algorithm (WOA) in [9] is proposed to solve the problem of resource distribution (RA) in the Internet of Things (IoT) with the aim of optimized RA and to reduce the total cost of connectivity between resources and gateways. Comparing other existing algorithms with the proposed algorithm, the findings show that the algorithm proposed works properly. In terms of the total cost of communication, the planned process is greater than others, depending on various parameters.

In the context of the sections referred to above, the main contributions of this paper are as follows:

(i) As mentioned earlier, the notion behind PACR is like a person writing his will on a deathbed before he dies. Similarly, when the energy of the sensor is less than the threshold, this sensor node is considered as an ill node on a deathbed. So, it will become a recovery coordinator and will draw up a recovery plan. It speeds up recovery by reducing the time taken to diagnose a failure, establishing a recovery coordinator and eventually creating a recovery plan

(ii) In addition to the accelerated recovery, PACR's other contribution is to make the node movement efficient. As a replacement for relocating the recovery sensor node to the exact location of the failed node, it can travel just the distance where it can get near the failed sensor node and be connected to its neighbor. This will reduce the movements of recovery nodes. This kind of recovery strategy plays a key role in saving energy in movement; it decreases the trip distance and the number of neighbors participating in the recovery

The rest of the paper is organized as follows. Section 2 gives the literature review work in a summarized manner, 
whereas Section 3 has the details of the proposed algorithm. Validation of the proposed algorithm by outcomes of simulations is presented in Section 4, whereas Section 5 concludes the paper and discussion of future work.

\section{Related Work}

This section includes a survey on the recent work done in the domain of coverage and connectivity restoration issues in WSNs. The survey demonstrates that recovery policies opted by researchers in this regard broadly focus on sustaining network connectivity while maintaining prefailure network topology. The restoration policies work in two main steps: first is the detection of failure node and second is the implementation of the recovery plan.

In [10], the authors proposed an algorithm under the name of Partition Detection and Recovery Algorithm (PADRA) which predetermines the critical nodes that can potentially segment the network. After the identification of cut vertexes, a suitable neighbor is appointed as a recovery node. When such a node fails, the selected neighbor replaces it whose relocation does not break the network into disjoint regions. Cascaded movement is initiated by such repositioning which consumes a lot of energy.

In [11], the authors proposed an algorithm under the name of Coverage Conscious Connectivity Restoration (C3R). The technique assures connectivity restoration while maintaining the prefailure network coverage by temporarily relocating the neighboring node at the position of the failed node. After the detection of failure, a schedule is generated by a recovery coordinator which holds the information about the participating neighboring node in the recovery process along with their turns. Each node on its predefined turn will be relocated at the location of the failed node, and after staying there for some time, it will be sent to its original position. After that, the other node on the schedule list will be repositioned to the failed node, and it will repeat the same process. The adopted back and forth movement will restore connectivity but at the cost of more energy loss and message overhead.

In [12], the authors proposed the technique Volunteerinstigated Connectivity Restoration (VCR) which is aimed at limiting the cascaded movement of nodes. The failure of a node like any other protocol is detected through missing heartbeat signals. The nearest neighbor of the failed node is selected for the recovery based on the vicinity, as only the closest neighbor performs the restoration which aids in restricting the movement of all the neighbors towards the failed node. The approach might be helpful in the case of a static environment; however, there is no guarantee of restricting cascaded movement in a dynamic environment. Another limitation of VCR is the selection of the nearest neighbor for recovery irrespective of its energy level which might end up choosing a neighbor with a low energy level which will eventually lead to further node failure and reducing the life of the network.

In [13], the authors proposed an algorithm that focuses on two issues: first is the connectivity restoration by node repositioning and second is the reduction in coverage holes created by node relocation which is named as the Minimum Movement Technique (MMT). First of all, the critical nodes of the network are identified using an algorithm named cut vertex determination. After the identification of the cut vertex, these cut vertexes will allocate a node from its neighbor to replace it in case of failure and it will be called dominant. In case of failure, the cascaded movement takes place starting from the dominant position until it reaches the failed node. MMT is used to reduce the coverage holes caused by cascaded movement by placing the failure handler close to the failed node without losing its connectivity.

In [14], the authors proposed a connectivity restoration algorithm called Least-Disruptive topology Repair (LeDiR) for WSANs. The failure of a node is identified through a missing heartbeat signal. In case of a node failure, each neighbor based on the routing table will define the suitable measures to be taken and their role in it. However, in case of critical node failure, LeDiR is aimed at finding out the smallest block; therefore, only neighbors belonging to the smallest block will participate in the recovery plan. The repositioning of the smallest block encourages the relocation of a minimum number of nodes which will reduce the overhead along with the guarantee of not extending lengths of paths between nodes. LeDir exploits already existing activities of routing and hence does not add on any communication overhead.

In [15], the authors presented an approach called RAM which extends the DCR algorithm. The technique focuses on handling multiple node failures simultaneously. The objective is achieved by selecting a backup node that is noncritical, and it can have only one primary. Another constraint in this regard was that two inline critical actors cannot serve as each other's backup. Finally, if a critical node selects a noncritical node as a backup, the backup node also has to select a backup node for itself to handle multiple adjacent node failures.

In [16], the authors present a technique called Incremental Optimization based on Delaunay Triangulation (IO-DT) which is a relay node placement issue. The algorithm solves three-terminal problems and identifies the least number of relay nodes by making use of subsets of terminals. The objective is accomplished by calculating the Delaunay triangulation (DT) of terminals, and it is repeated on the triangles. Consider this, if choosing this triangle to reduce the number of relay nodes as compared to most-based solution then it will transform the triangle into final topology. The disadvantage of such an approach is its complexity.

In [17], the authors proposed an algorithm that is aimed at controlling energy utilization and also reconnecting the network in case of failure of a node. The methodology makes use of a multichannel to communicate so that they can reduce the number of neighboring nodes sharing the same channel which aids in reducing the collision. In case of a node failure, the network performs the connectivity, and the restoration plan is executed in two steps. The first step involves reorganizing the nodes to reconnect the lost connection created due to the failure of a node. The second step is to recolor the repositioned nodes without disturbing the entire network. Performance evaluation is done using simulation. 
In [18], the authors proposed a technique called Optimal Relay Node Placement (ORNP) for the restoration of lost connectivity while effectively reducing the number of relay nodes in WSNs. ORNP segments the entire region of interest into subregions by using the Voronoi partition. Each segment is the Voronoi cell which consists of cluster heads along with their cluster members. When the network gets partitioned, a node sharing two or more cluster heads will become a relay node. The assigned task of this node is to link broken segments. Furthermore, 3-star technology will reduce the number of disjoint regions by repeatedly deploying a relay node in each of the three disconnected regions. Lastly, all the leftover disjoint regions are identified by calculating the center of mass. The connectivity of a network is restored by relocating the relay nodes which are located towards the center of mass.

In [19], the authors proposed a technique called Recovery through Inward Motion (RIM) to deal with connectivity gaps created due to node failure. The missing hello messages provide information about the failure of the node. After the detection of failure, all the neighboring nodes start in an inward motion towards the failed node, hence participating in the recovery process. Although RIM is a noncomplex approach, cascaded relocation of the neighboring nodes towards failed nodes results in two major drawbacks. Firstly, the movement will cause a reduction in the coverage area, and secondly, it will lead to more energy consumption which might decrease the lifespan of the network.

In [20], the authors devise a technique to develop a path from the region of interest to the base station with the focus of encountering the least number of hops. It will enable us to find out a minimum distance in such a way that the network remains connected. Their opted strategy comprises of two other algorithms. The first algorithm will search for the robot which is at the minimum distance to the required location, and then it will be allocated to that area. Afterward, the algorithm looks for the closest disjoint robot and directs it in the transmission range of a robot that is already linked with the assigned robot. The process is repeated to rejoin the whole network. In the second algorithm, the goal is to find out those areas where it can optimally deduct the number of hops from the base station to the location of the event.

In [21], the authors aim to devise a technique to deal with the issue of connectivity restoration while taking into consideration the significance of the affected area. The strategy is based on relocating the nodes to the area of high importance while rejoining the detached clusters. The algorithm is built upon a round table technique in which a negotiator from each cluster travels and negotiates with others by interchanging information and determining the path for reconnection. In addition to the basic round table, the proposed technique also replaces the failed nodes. Recovery and reconnection are implemented in three modules: reconnection, recover and reconnect, and, lastly, reconnect and recover.

In [22], the authors proposed an algorithm for connected p-percent coverage problems in Wireless Sensor Networks (WSNs) to boost overall network life. In this work, they investigate the p-percent coverage problem (PCP) in WSNs that need p-percent of the region to be monitored correctly and to find out any additional criteria for the related ppercent coverage problem. They suggest a pDCDS algorithm that is a PCP-based learning automation algorithm. PDCDS is a degree-restricted connected dominating set-based algorithm that detects the minimum number of nodes to control the field. The simulation results show that pDCDS can dramatically improve the existence of the network.

In [23], the authors presented PCLA, an efficient learning automation algorithm that is aimed at reducing the number of sensors to be enabled, so that a certain portion of the area of interest is protected and the communication between the sensors is maintained. Simulation results show how PCLA can efficiently pick sensors to meet the constraints imposed, thus ensuring better output in terms of both the working-node ratio and the WSN lifetime. They also demonstrate how PCLA outperforms state-of-the-art partial-cover algorithms.

In [24], the authors suggest an abnormal cellular learning automation- (ICLA-) based algorithm, called SPLA, to maintain the safety of sensors. The goal of learning automation at each ICLA cell with proper rules is to investigate the minimum possible number of nodes to guarantee the selfprotection requirements of the network. To test the efficiency of SPLA, several simulation experiments have been carried out, and the results obtained show that SPLA performs on average 50 percent better than the maximum independent set and the minimum connected dominant set algorithms in terms of the active node ratio and can provide a twofold reduction in energy consumption. Table 1 shows the characteristics of different techniques.

The above approaches for solving the connectivity problem have been suggested in the literature. While all these strategies have the potential to regain connectivity, these techniques do not address the coverage problem. Only C3R [7] gives a solution for connectivity and coverage. C3R gives the solution, by moving neighboring nodes towards the direction of the failed node. This motion can result in energy dissipation. Besides, after the diagnosis of failure, the recovery process will begin, which will introduce a time delay, rendering it ineffective for mission-critical scenarios. By proposing a novel Position-Aware protocol for Connectivity Restoration (PACR) algorithm, we aim to fill these loopholes. In our technique, if a sensor node's energy is less than a threshold, it will become a recovery coordinator and will establish a recovery strategy. By taking out the time required to diagnose a loss, making a recovery coordinator, and eventually generating a recovery plan, it makes the recovery fast.

The other strength of PACR, in addition to the quick recovery, is to make the network energy efficient. First of all, instead of pushing the recovery sensor to the exact location of the failed node, it can just travel that much distance where it can get close to the failed sensor and become linked to its neighbor. Along with it, if there is a sensor that can be repositioned without losing any connections of its own, and in this situation, only the neighbors can do the recovery in touch with neighbors of the failed node. This design of the recovery strategy plays a critical role in reducing the resources involved during transportation by limiting the 


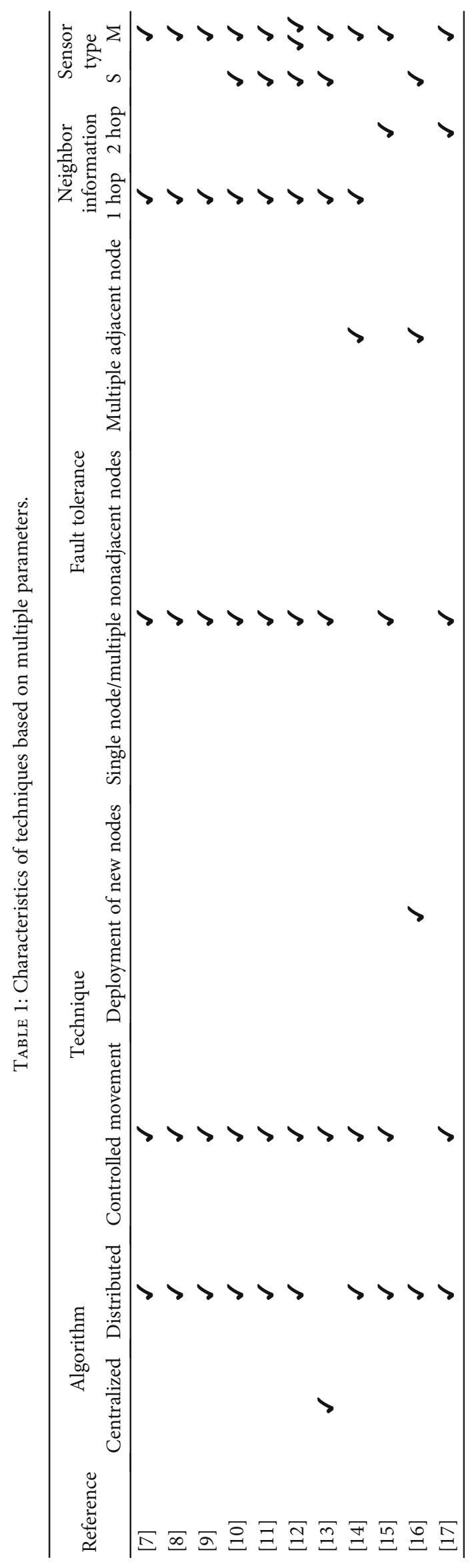




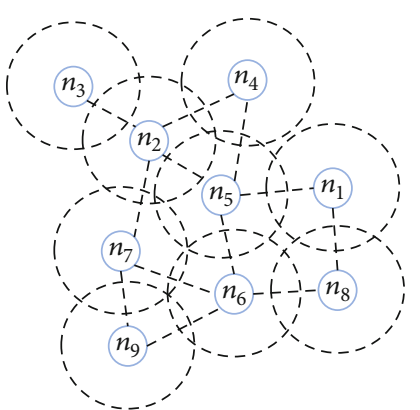

(a)

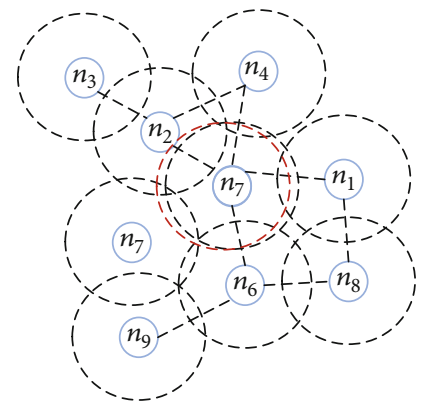

(d)

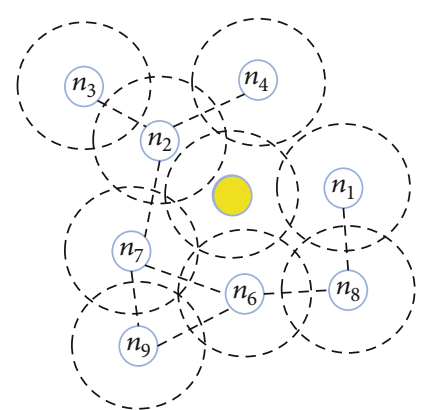

(b)

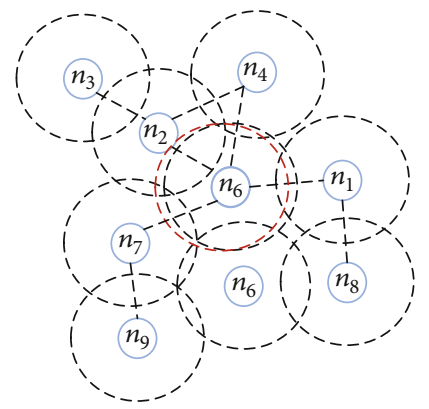

(e)

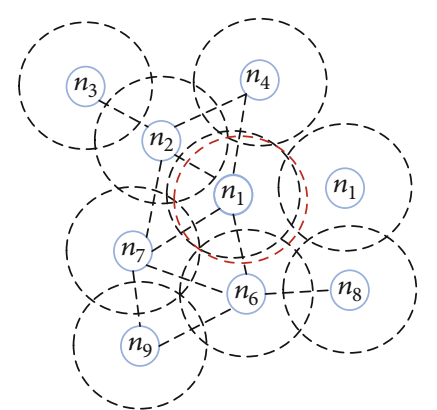

(c)

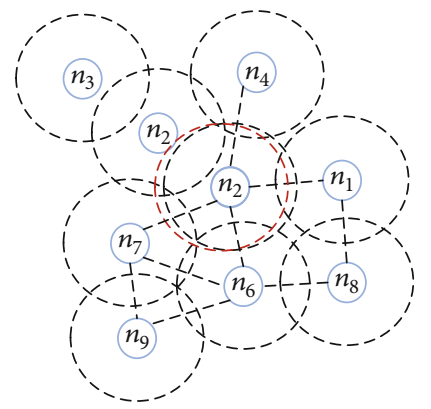

(f)

FIGURE 1: (a) PACR; (b) $n_{5}$ energy running low; (c) $n_{1}$ changes its position; (d) $n_{7}$ changes its position; (e) $n_{6}$ changes its position; (f) $n_{2}$ changes its position.

distance of travel and the number of neighbors participating in the recovery process.

\section{System Model and Problem Formulation of PACR}

PACR is true for a network of mobile sensor nodes. Mobile nodes in a hierarchical network architecture can be part of a flat network topology or form a second level, such as a community of actors or aggregation and forwarding units. Sensors in a field of interest are deployed randomly. In the network, no spare nodes are given, however, whether either there is a node harm coverage void in the network or the number of active nodes is below three nodes. We consider, however, only one loss at a time. PACR does not cope with node errors that are overlapping.

Separate sensing and communication ranges for a node may occur. No relation is presumed between the two ranges. It is believed that during network booting, trap nodes discover each other and form a connected network [7]. To plug a void in coverage and/or address a connection error, a node is believed to be able to travel on request. While absolute anode position coordinates are not required, PACR assumes that its location can be determined by a mobile node relative to its neighbors [8]. Each node is believed to maintain a list of its direct (1-hop) neighbors.

The loss of a node due to malfunction may also impact network access, in addition to impacting network coverage. This paper focuses on retaining network connectivity when a node fails while ensuring coverage for prefailure. Consider the network topology seen in Figure 1(a), for instance. Nodes $n_{1}, n_{2}, n_{7}, n_{6}$, and $n_{8}$ are contiguous with $n_{5}$. The loss of $n_{6}$ would disconnect $n_{4}$ and $n_{8}$ from their neighbors from the rest of the network and leave a vacuum in coverage since no other node has its sensing spectrum overlapping with $n_{5}$. It just shifts the coverage void to another part of the sector, either in the inner part of the network or at the periphery, whereas replacing $n_{5}$ with another node would restore the connection. PACR opts to solve this problem by momentarily replacing the failing node with one or more of its neighbors. The concerned nodes switch back and forth so that the network topology and coverage remain largely identical to their prefailure status.

\section{Position-Aware Connectivity Restoration (PACR) Protocol}

4.1. Prefailure Operation. The only prefailure information needed by PACR for each node is to have a list of 1-hop neighbors. With each node transmitting a Hello message to introduce itself to its neighbors, this list is created postdeployment [7]. The node's location and the ID of all its neighbors will also be tabulated by each node. Absolute GPS-based coordinates are not required for the position; for PACR, relative positions will suffice. If there is a failed neighboring node, this information will be used. When a node A does not receive a preset number of Heartbeat messages from a neighboring node, $\mathrm{F}$, it assumes that $\mathrm{F}$ has failed. When a node moves, it forms its neighbors to avoid being wrongly interpreted as defective. When a node moves, it forms its neighbors to avoid being mistaken. In other words, each time one of them changes its location, the list of neighbors can be changed. 
Node A conducts the recovery process after detecting a neighbor $\mathrm{F}$ failure. It is important to remember that there are two general strategies. The first is to determine whether the absence of $\mathrm{F}$ would partition the network and respond only if $\mathrm{F}$ is a cut vertex. This option, adopted by [11], prevents overreacting to a failure that does not prevent nodes from accessing each other.

4.2. PACR Working. The proposed PACR makes use of mobile sensors and requires the nodes to maintain knowledge of their 2-hop neighbors. Each node will keep in check its energy level, and whenever it drops below a threshold, it will become a recovery coordinator and design the recovery plan. The major steps involved in PACR are as follows.

4.2.1. Status Table for 2-Hop Neighbor. In the postdeployment phase, each sensor will broadcast a Hello message to its immediate neighbors. After the introduction, every node must maintain a status table of its 2-hop neighbor to cater to the changes in the network topology. The parameters of the list involve \{Node_ID, Distance\}. The information about the distance of 2-hop neighbors will aid to reduce the energy consumption by restraining the far sensors from getting involved in the recovery process. When the energy of a sensor falls below a predefined value, then it will initiate its recovery plan.

4.2.2. Distance Measurement. In the proposed algorithm, recovery nodes will move a minimum distance so that they cover the connectivity and coverage of the failure node. So, for the recovery process, every recovery node will calculate the overlap area with its neighbor nodes and the minimum distance for the movement. Let $r_{0}$ be the coverage radius of $N_{0}$ and $N_{1}$ nodes and " $d$ " is the distance between nodes as shown in Figure 2(a). The maximum distance $N_{0}$ or $N_{1}$ can move is

$$
l_{1}=\frac{2 r_{0}-d}{2} .
$$

Now, for the calculation of the required distance for the coverage and connectivity of the failure node, each recovery node calculates the distance that it moves in the direction of the failure node to cover the connectivity. Each node has 2hop information, so it calculates the distance with the node which has a maximum distance from it. $d_{0}, d_{1}$, and $d_{2}$ in Figure 2(b) are the known distances for $\theta$.

$$
\sin (\theta)=\frac{d_{0} d_{2}}{d_{1}}
$$

For the required distance, $\theta$ and $d_{1}$ are known and $d_{r}$ is

$$
d_{r}=2 r_{0} \text {, }
$$

where $r_{0}$ is the radius of the coverage area. Now, the distance a node will move for the coverage and connectivity is

$$
d_{n}=d_{0}-\frac{d_{1} * d_{2}}{\sin (\theta)}
$$

4.2.3. Crafting Recovery Plan. As mentioned above, when the energy level of the sensor is less than 70\% of its initial energy value, it will become a recovery coordinator, and its charge now is to identify the most competent backup neighbors. It will shortlist five of the neighbors with the least distance and with their energy above $30 \%$. Afterward, a ranking list will be generated based on overlapping coverage, distance, and energy level of the node. This list will be holding the information about the number on which each node will be relocated along with the distance it has to travel to get near the failing node. After the list is broadcasted to the backup neighbors, each participating neighbor will examine its demanded repositioning distance. If a node is in the admissible moving range while staying connected to its neighbor, in this case, only that node will be the backup node. This message will be broadcasted to the failing node which in return will inform the other recovery neighbors by a broadcasting message about a change in the recovery plan. If two of such nodes are available, then the one with the higher energy will be selected. In case no node has overlapping coverage, then recovery will be done according to the set ranking list. Another important case to study will be the sudden failure of a node. Here, we will exploit the information about the 2-hop neighbor. As each surrounding neighbor has the information about the distance of two neighbors, so the one with the least distance will become a recovery coordinator again restraining the movement of all neighbors and eventually saving the energy of the network.

4.2.4. Illustrative Example. The operation of PACR is demonstrated under consideration of the network topology in Figure 1 . The scenario of the failure of $n_{5}$ node is shown in Figure 1. The following steps will be done.

(1) This failure is detected by the neighboring nodes of $n_{5}$

(2) According to the ranked list, the first one for recovery in the illustration is $n_{1}$ being the closest

(3) Before repositioning, it will broadcast the message to its neighbors to avoid its incorrect assumption of failure

(4) Another point to notice here is that the original position of $n_{5}$ is shown in the red circle whereas the new position of the recovery node $n_{1}$ is in blue to explain the phenomenon that $n_{1}$ will not be relocated at the exact position of $n_{5}$

(5) After being there for its allocated time, it will move back to its original position and all the backup nodes will repeat the same procedure as $n_{1}$

(6) Figure 3 illustrates the scenario when the coverage hole can be filled by a slight repositioning of a single node

(7) After the detection of $n_{5}$ failure, $n_{2}$ will be the only relocated backup node staying in contact with its neighbors and making new contacts with the neighbors of the failed node $n_{5}$

The next section will discuss the pseudocode of PACR. The pseudocode will describe how a single node will tackle the following situations by using PACR 


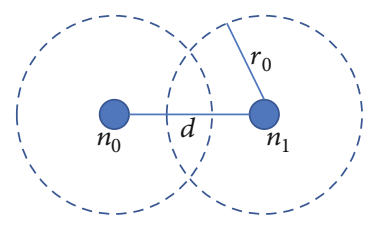

(a)

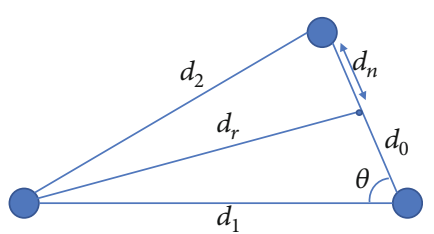

(b)

FIgURE 2: (a) Overlapped region of nodes $n_{o}$ and $n_{1}$; (b) illustration of the calculation of the required distance.

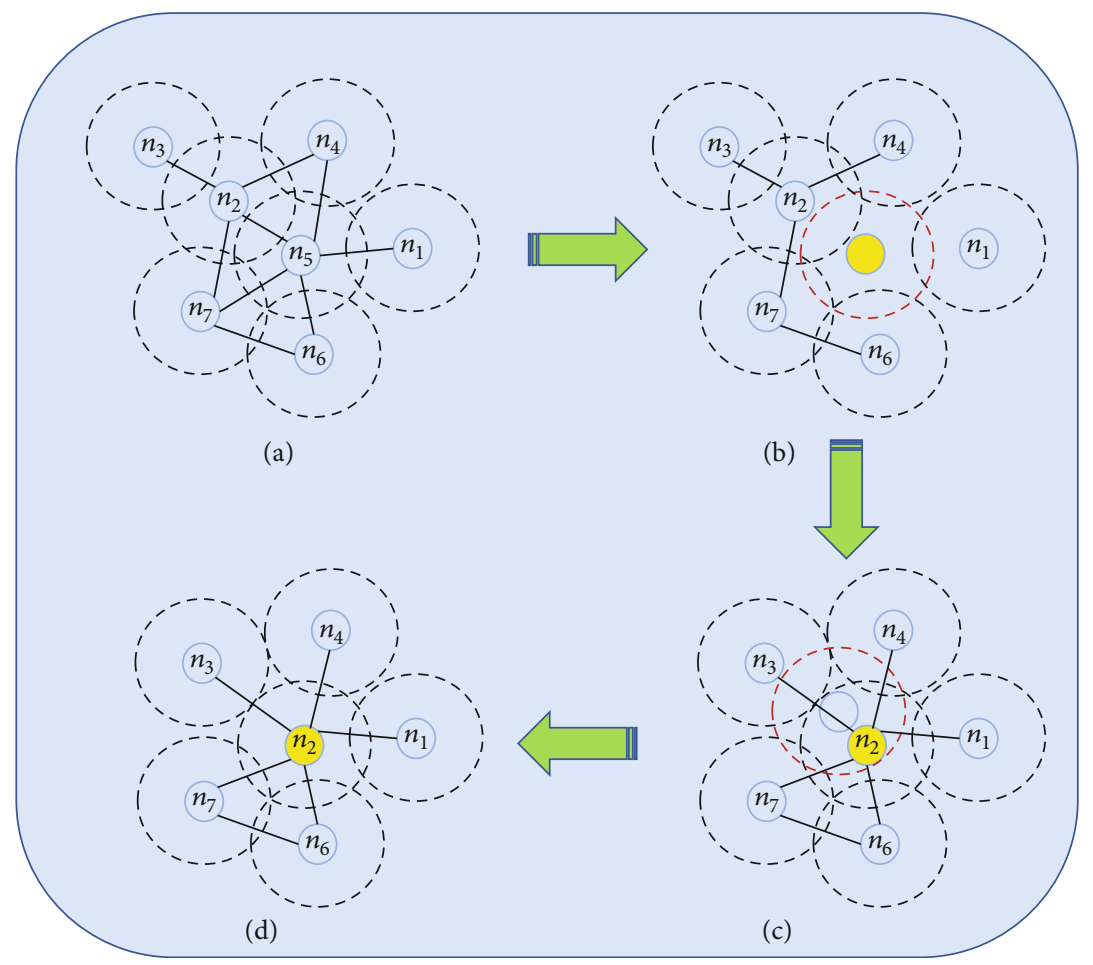

FIgure 3: (a) PACR one node recovery; (b) $n_{5}$ energy running low; (c) $n_{2}$ permanent relocation; (d) new topology.

(1) when a node has less than 70\% energy (coordinator)

(2) when it becomes a recovery node

4.3. Complexity Analysis. PACR's time complexity depends on the number of slave keepers, sensor nodes, and the energy measurement process for each node of the sensor.

The number of coordinators is represented by $c$, the number of nodes of the sensor connected to each coordinator is represented by $n$, and the energy measurement method for each node of a sensor is represented by $E$.

After the coordinator sends the signal to the recovery sensor nodes, the signal is sent to the sensor nodes involved. Now residual energies are correlated with threshold energy by sensor nodes. If each recovery sensor node has sufficient resources, the total number of iterations (best score) required to run the algorithm is $(n * E)$. If the number of recovery sensor nodes is $c$, then $\{(n * E) * c\}$ would be the total executions needed. In the worst case, if each sensor node's energy is less than the threshold energy, $\{(n * E) *(n-1)\} * c$ is the total number of iterations (worst score) required to execute the algorithm.
The essence of our proposed algorithm is polynomial. For each slave keeper, the external loop executes, and for each sensor node allocated to the new slave keeper, the nested loop is executed. The complexity of Algorithm 1 is $\mathrm{O}\left(n^{2}\right)$ in terms of the Big-O notation.

4.4. Energy Model. An energy model that is used for this research article is presented in [23]. A " $B$ " bit data packet over distance " $d$ " is transmitted and received by the sensor nodes. The energy expended per bit by the receiver is given by (5). The residual energy can be calculated by Equation (7).

$$
\begin{aligned}
E_{\mathrm{Tx}}(B, d) & =\left\{\begin{array}{cc}
\left(E_{\text {elec }}+\varepsilon_{\mathrm{fs}} d^{2}\right) B & d<d_{0}, \\
\left(E_{\mathrm{elec}}+\varepsilon_{\mathrm{mp}} d^{4}\right) B & d \geq d_{0},
\end{array}\right. \\
E_{\mathrm{Rx}}(B) & =E_{\mathrm{Rx}-\mathrm{elec}} B, \\
E_{\mathrm{reng}}(n) & =E_{\mathrm{max}}-E_{\mathrm{Tx}}(B, d)-E_{\mathrm{Rx}}(B) .
\end{aligned}
$$

The terms used in this energy model are explained in Table 2. 


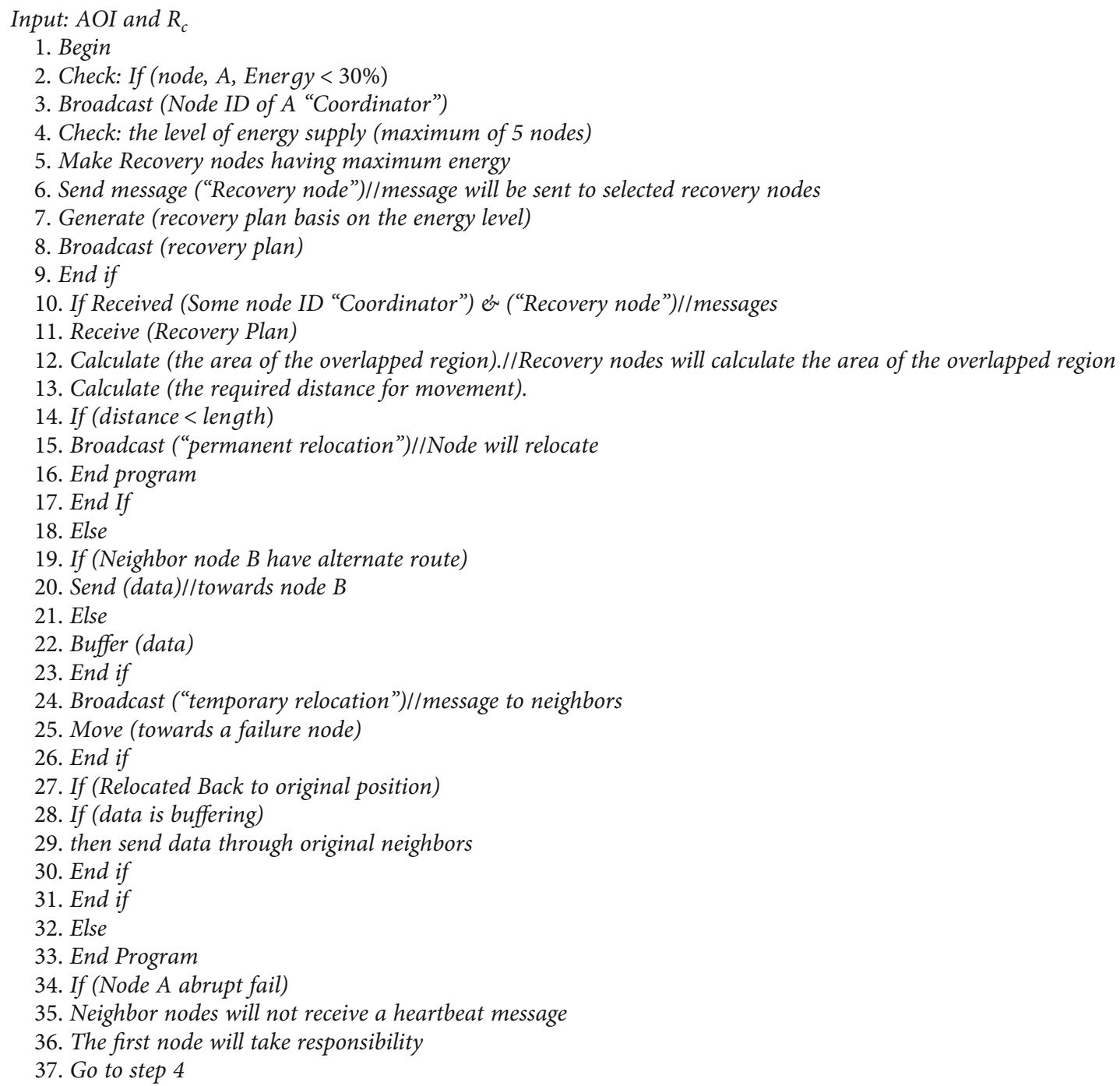

Algorithm 1: PACR algorithm.

TABLE 2: Different variables used in the energy model.

\begin{tabular}{lc}
\hline Variable & Definition \\
\hline$E_{\mathrm{Tx}}$ & Energy consumption to transmit data \\
$E_{\mathrm{Rx}}$ & Energy consumption to receive data \\
$E_{\mathrm{reng}}$ & Residual energy \\
$E_{\text {elec }}$ & Energy consumed by a transmitter per bit \\
$E_{\mathrm{max}}$ & The initial energy of nodes (maximum energy) \\
$E_{\mathrm{Rx} \text {-elec }}$ & Energy consumed by a receiver per bit \\
$\varepsilon_{\mathrm{fs}}$ & The energy required by an amplifier in free space \\
$\varepsilon_{\mathrm{mp}}$ & The energy required by an amplifier in multipath \\
$d_{0}$ & Threshold distance for sensor nodes \\
\hline
\end{tabular}

\section{Simulation Setup}

The OMNeT++ platform is used for all the simulations. A comparative analysis is done between Survivability-Aware Connectivity Restoration (SACR) and Connected Dominating Set (CDS) algorithm [21, 22].

5.1. Settings of Simulation. In the experiments, to form WSNs, a set of mobile sensor nodes are initially randomly placed in a $900 \times 900 \mathrm{~m}^{2}$ area. The communication and sensing ranges of all nodes are assumed to be the same. Performance is evaluated by the following metrics.

5.1.1. Total Distance Moved. This records the cumulative distance jointly traveled after recuperation by the nodes concerned. This can be envisaged as a networkwide review of the success of the recovery schemes used. The optimization formulation opts to find the best length and relocation frequency for each sensor node $s_{i} \in S$, where $i=1,2, \cdots \cdots N$ 
number of sensor nodes present in set $S$. Assume that node A is at a distance from failed node F. Suppose A will travel at a constant speed, Sp. Therefore, during one migration, the time and distance that node A will take to switch back and forth to $\mathrm{F}$ is provided by

$$
\begin{gathered}
\tau=2 * \frac{\text { distance }_{\mathrm{A}}}{\mathrm{Sp}}, \\
\text { distance }_{\mathrm{A}}=\frac{\tau * \mathrm{Sp}}{2} .
\end{gathered}
$$

5.1.2. No. of Nodes Relocated. This tracks the number of mobile nodes $(M)$ that have been shifted during recovery. This metric tests the scope of the method of repairing network links.

5.1.3. No. of Exchanged Messages. This records the cumulative number of exchanged messages (EM) between the nodes. This calculation tests the communication overhead imposed by the recovery process.

5.1.4. Communication Range $\left(R_{c}\right)$. All nodes of the experiment have the same communication range. The importance of $R_{c}$ affects the initial WSN topology. Although a small $R_{c}$ provides a sparse topology, a large $R_{c}$ improves overall network connectivity. In strongly connected WSNs, very few nodes need to be included in the cascaded movement, and the reconstruction process would converge easily with little overhead; a big $R_{c}$ value will increase the number of failed node neighbors and thus increase the overhead experienced by these neighbors.

5.1.5. The Network Lifetime. This metric compares the time for the first rehabilitation participant to run out of energy. From the beginning of the network to the first sensor node, the network lifetime $\left(N_{L}\right)$ is measured as the number of tours carried out by the mobile node, such that its energy is completely dissipated. The network lifetime can be computed as

$$
N_{L}=\frac{E_{\text {initial }}}{\lambda},
$$

where $E_{\text {initial }}$ is the initial energy and $\lambda$ is the maximum energy consumed by a node

$$
\lambda=\max _{i \in S}\left(E_{i}\right)
$$

where $i=1,2, \cdots \cdots N$.

5.1.6. Sensing Range $\left(R_{s}\right)$. This impacts the coverage of the node. Short ranges make PACR a favorable framework for recovery. If $\psi$ denotes the number of migration rounds made by a node $\mathrm{A}$ to $\mathrm{F}, \mathrm{ab}_{\mathrm{A}}$ denotes the cumulative time $\mathrm{A}$ spends away from its home area, letting " $t$ " be the time node that A spends at $\mathrm{F}$ over one round and $\mathrm{Pre}_{\mathrm{A}}$ be the time it spends at its original site. Therefore, $\mathrm{ab}_{\mathrm{A}}$ can be calculated as

$$
\mathrm{ab}_{\mathrm{A}}=\psi+\mathrm{t}+\tau
$$

TABle 3: Simulation setup.

\begin{tabular}{lc}
\hline Simulation parameters & Values \\
\hline Area of simulation & $900 \times 900 \mathrm{~m}^{2}$ \\
No. of nodes & $50 \sim 250$ \\
Size of the data packet & $800 \mathrm{bits}$ \\
$E_{\text {elec }}$ & 70 nanojoule $/ \mathrm{bit}$ \\
$E_{\mathrm{max}}$ & $50 \mathrm{~J}$ \\
$\varepsilon_{\mathrm{fs}}$ & 30 picojoule $/ \mathrm{bit} / \mathrm{m}^{2}$ \\
$\varepsilon_{\mathrm{mp}}$ & 0.0015 picojoule $/ \mathrm{bit} / \mathrm{m}^{2}$ \\
$d_{0}$ & $80 \mathrm{~m}$ \\
$R_{c}$ & $25-150 \mathrm{~m}$ \\
Simulation tool & OMNeT ++ \\
\hline
\end{tabular}

The goal of the optimization model is to maximize $\mathrm{F}$ coverage, i.e., to maximize the time spent by all replacements covering the $\mathrm{F}$ region.

$$
\operatorname{Max} \sum_{A \in S} \mathrm{t} * \psi
$$

5.1.7. Throughput. Throughput is the degree of efficient transmission of messages over a medium of communication. It is calculated as the ratio of the number of messages sent by $S_{i}$ $=\left\{s_{1} s_{2}, \cdots \cdots s_{N}\right\}$, where $N$ is number of nodes present in $S_{i}$ to $S_{j}$, and the number of messages received by $S_{j}=\left\{s_{1} s_{2}, \cdots\right.$ $\left.\cdots s_{M}\right\}$, where $M$ is number of nodes present in $S_{j}$ from $S_{i}$. Here, $M=N, S_{i} \in S$ and $S_{j} \in S$. Throughput is given as

$$
\text { Throughput }=\frac{\text { No. of messages send by } S_{i} \text { to } S_{j}}{\text { No. of messages recived by } S_{j} \text { from } S_{i}} .
$$

Given the fixed scale of the deployment area, the number of nodes in the network will influence the node density. The low node density reduces the coverage and considers our methods indispensable.

At first, the OMNeT++ simulator is designed for distributed systems. Then, simulation artifacts are generated (sensor nodes). By exchanging signals, virtual objects communicate with each other at distinct moments. For these simulations, the environment is $\mathrm{OMNeT}++$. Simulations are independently performed for each algorithm in $\mathrm{OMNeT}++$, and their data is then imported for plotting in MATLAB. Within 10 percent of the basic average, all simulation outcomes are taken, whereas for other simulations, the confidence analysis interval is taken as $90 \%$. The following steps are followed to test the efficiency of the proposed and baseline algorithms:

The success of the proposed PACR is validated through simulations carried in $\mathrm{OMNeT}++$. The simulation setup, performance metrics, and results are presented in this section. Table 3 gives details of the simulation setup. 


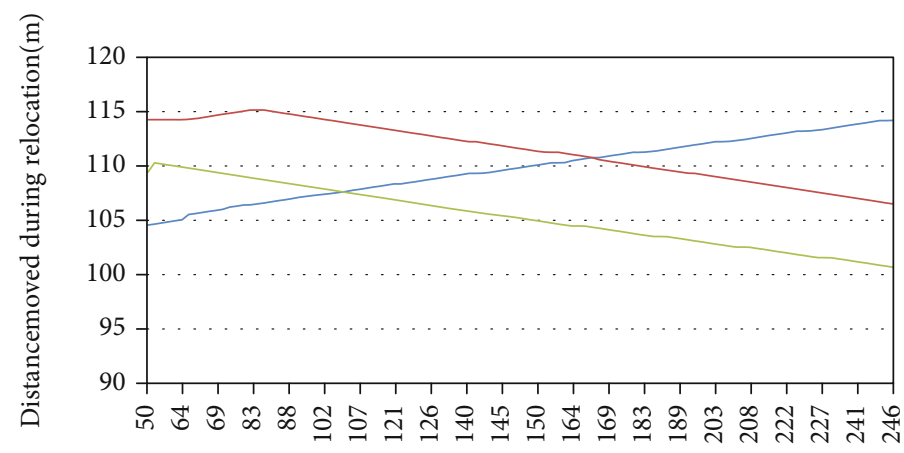

No. of nodes

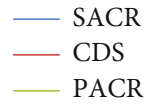

FIGURE 4: The total distance of all nodes in meters vs. distance moved during relocation.

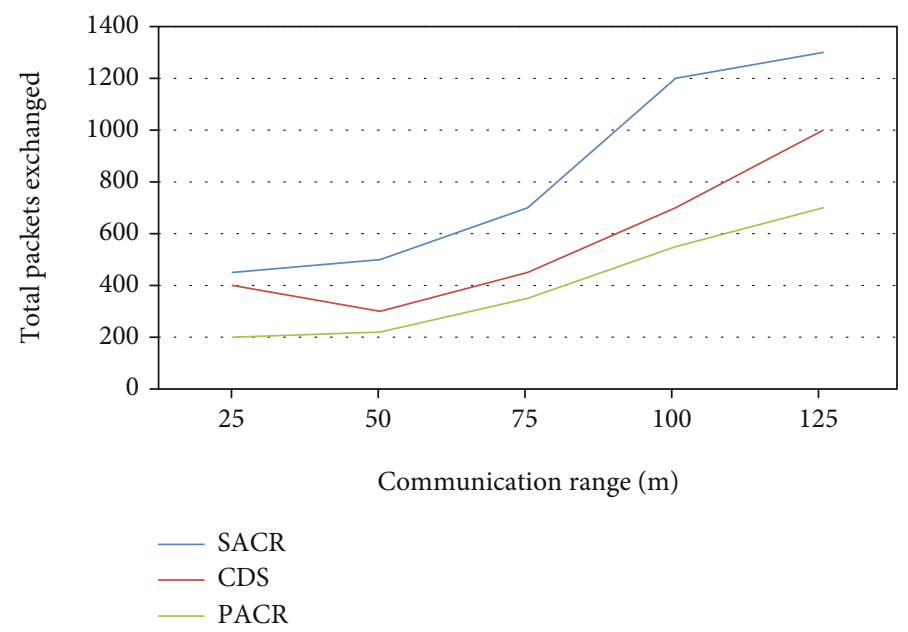

FIGURE 5: Total packet exchanged vs. communication range.

\section{Results and Discussion}

The distance that the nodes moved during relocation is shown in Figure 4. The distance a node should move depends upon the distance of one node to another node. There is an increase in the communication range as there was an increase in the distance of sensor nodes. This was not good for SACR and CDS because the increase in the distance is very rapid. When SACR and CDS are compared with the proposed PACR, the sensor node participation is limited in the recovery process in the case of PACR. The reason is only some neighbors of the failed sensor nodes take part. Cascaded relocation is avoided in PACR, which is initiated in SACR and CDS. The proposed technique must show the progress in the connectivity of the WSNs very well.

The received and delivered packets that were exchanged during the restoration of connectivity using SACR, CDS, and PACR methods are presented in Figure 5. The single broadcast message is used for these three techniques. In PACR, the messaging overhead is very less. In SACR, packets exchanged are maximum. The reason behind lesser message overhead in the proposed technique is that only neighbor nodes are involved in the recovery of the failed node, whereas in SACR and CDS techniques, the number of nodes that were relocated for the recovery process needs messages for their coordination.

During the recovery process, the average number of nodes moved to the number of nodes of said techniques is shown in Figure 6. The total distance travelled will increase with the increase in relocating nodes for these techniques. From Figure 6, the result shows that the node relocation of SACR is greater than the CDS technique. However, for the proposed technique, there is a smaller number of node relocation. The reason is because the proposed algorithm limits the recovery process to specific neighbor nodes.

In these figures, the impact of the number of nodes relocated in the said techniques on communication and sensing ranges is presented in Figures 7 and 8. The overall impact on communication and sensing ranges is limited reasonably by our proposed algorithm. Furthermore, for the networks that are not so dense, which are evenly distributed, the overlap ranges are at their minimal level for the proposed PACR. 


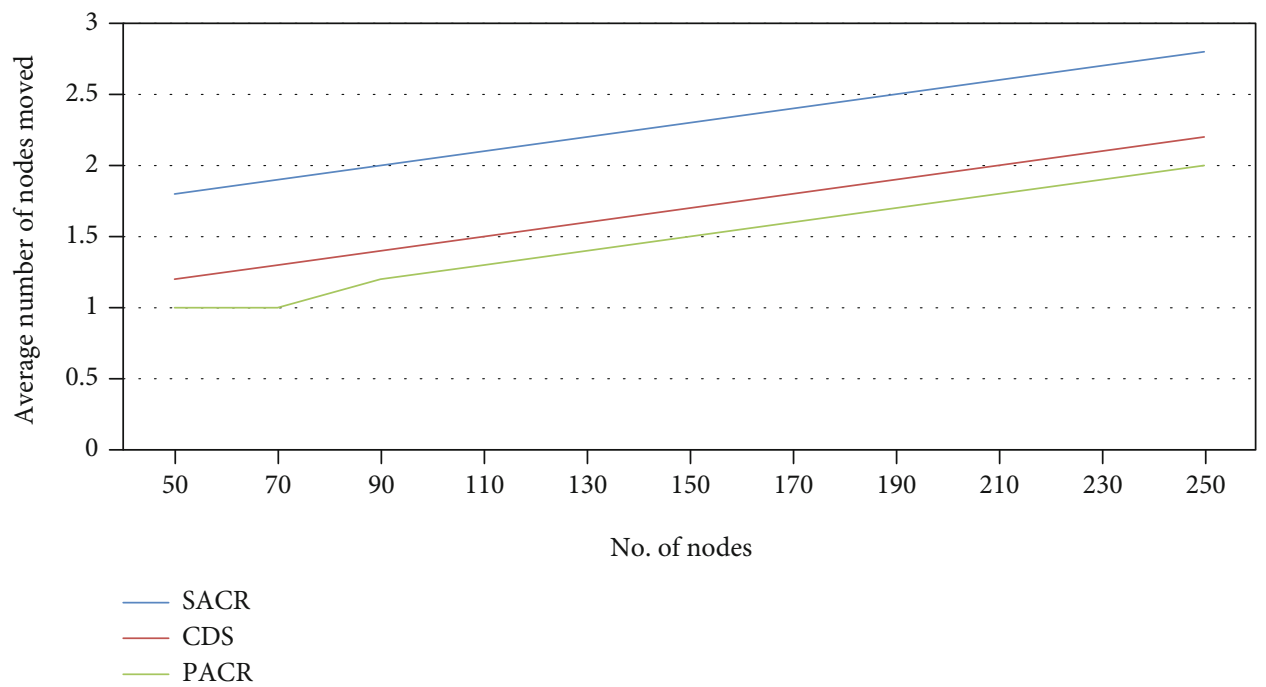

Figure 6: The average number of nodes moved vs. number of nodes.

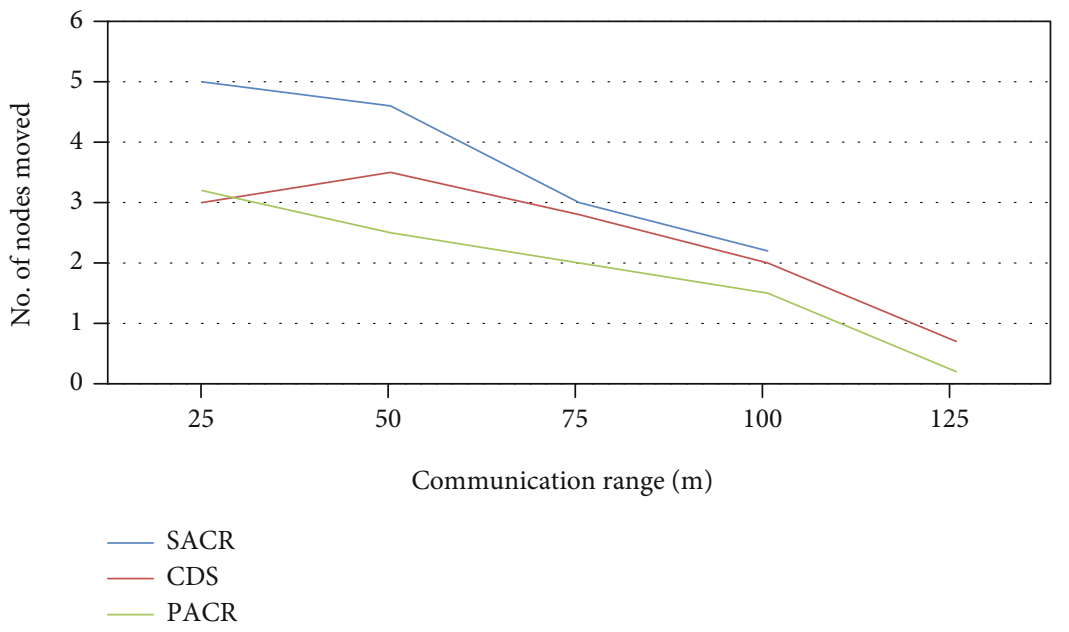

Figure 7: Number of nodes moved vs communication range.

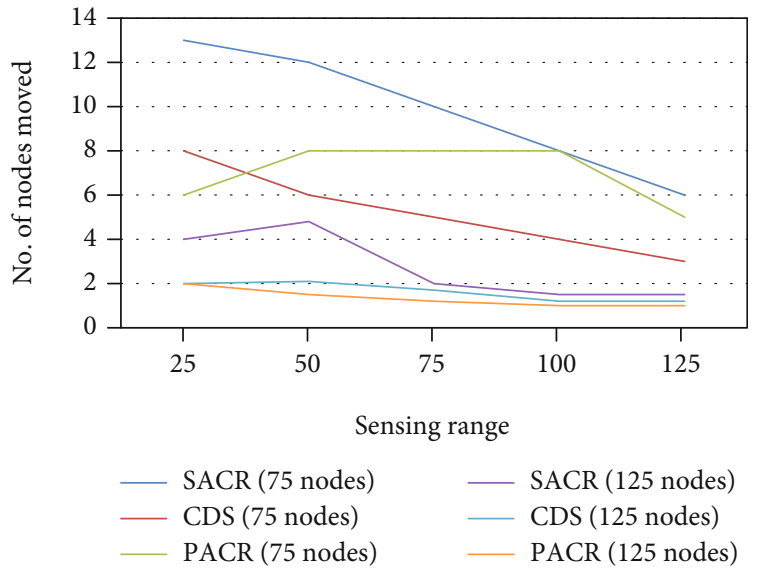

Figure 8: Number of nodes moved vs sensing ranges.
A greater increase in communication and sensing ranges was observed in SCAR.

For various communication and sensing ranges, the percentage reduction of the field coverage is shown in Figure 9. When $R_{s}$ is dominated by $R_{c}$, reasonable coverage reduction is perceived, because longer distances are needed for recovery nodes to travel between their original position and the position of the failed node.

The efficiency comparisons between the proposed PACR, SACR, and CDS protocols are shown in Figure 10. The total lifetime is increased by reducing the overhead of protocols in the network. The proposed protocol, therefore, has better performance in enhancing the lifetime of the network than the other baseline protocols. The main reason is that there is a perfect recovery plan for the neighbors of failed nodes, as only limited neighbors are given the role to recover the connectivity and coverage of failed nodes and there is a restriction on neighboring nodes that their energy must be $70 \%$. So, the chances of 


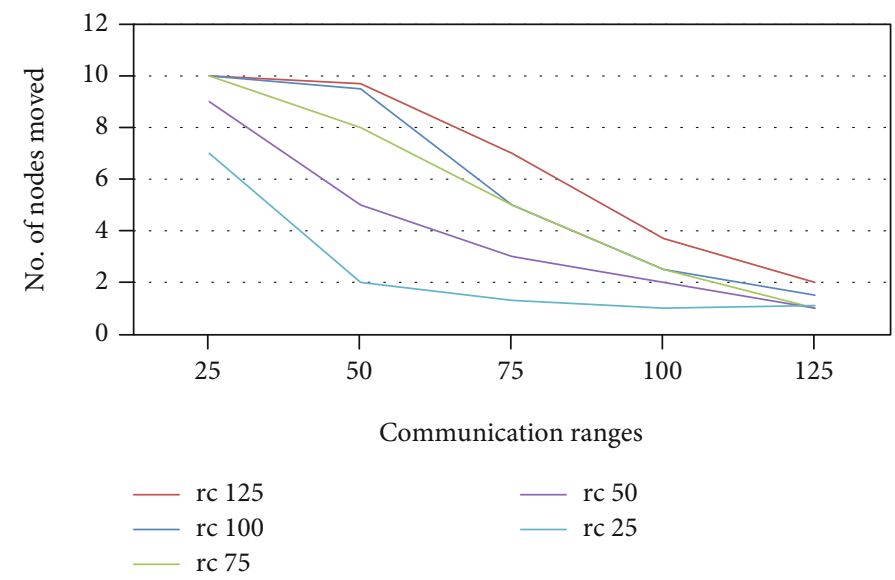

FIGURE 9: Number of nodes moved vs. communication ranges.

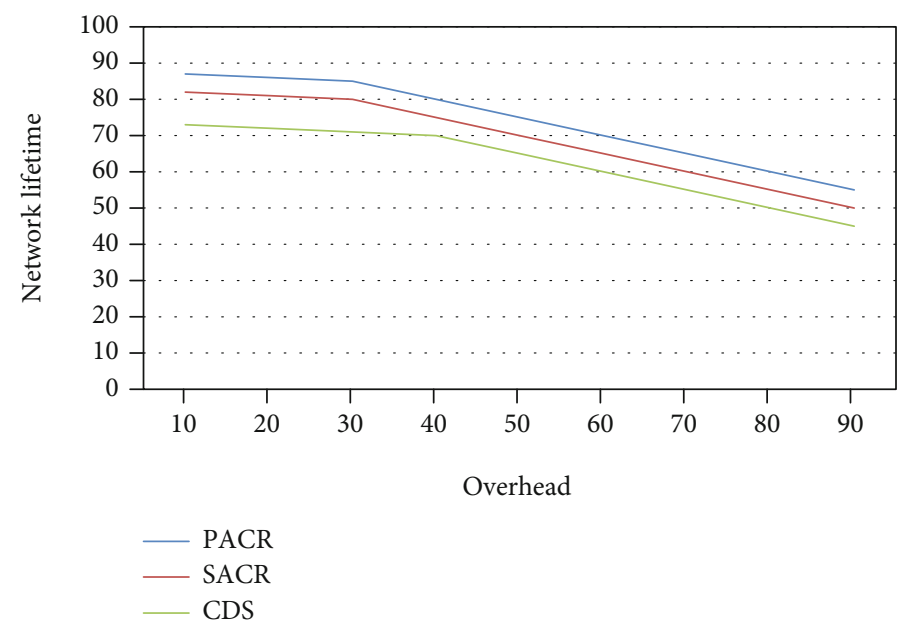

FIGURE 10: Overhead vs. network lifetime of Wireless Sensor Network.

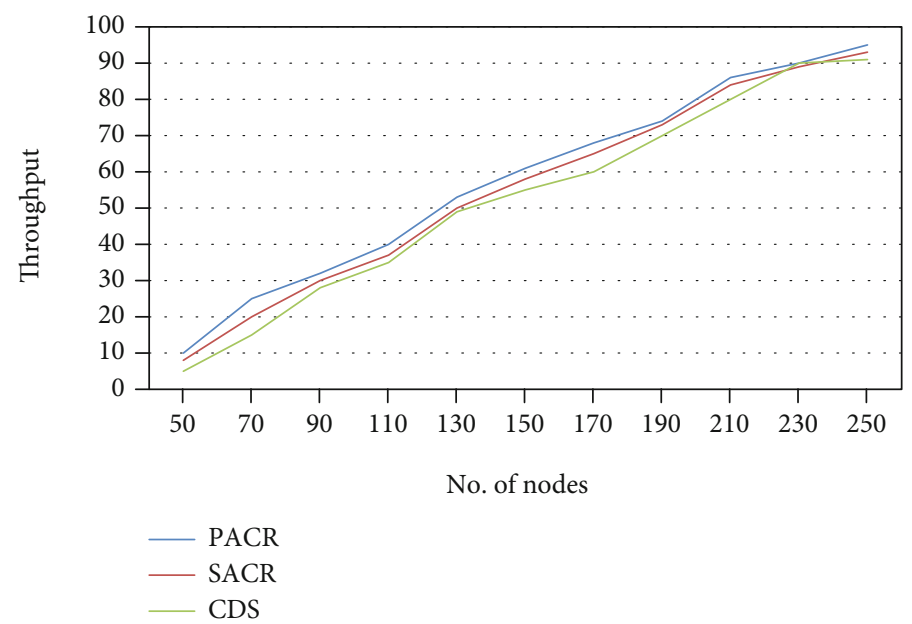

Figure 11: No. of nodes vs. throughput.

neighboring nodes participating in the recovery process to die sooner are lesser as compared to those of baseline approaches.
Figure 11 indicates that the proposed PACR is upgraded relative to the baseline algorithms. The number of nodes increases, which also increases the throughput. 


\section{Conclusions}

A novel algorithm known as the Position-Aware Connectivity is presented in this paper. The PACR resolves not only connectivity issues but also the issue of coverage. The proposed algorithm offers a solution in such a way that it does not permanently relocate the nodes. For recovery of the failure node, only neighboring nodes are responsible for the proposed technique. Selected neighboring nodes will make the restoration process very rapid as compared to other approaches. After spending their due duration of time at the position of the failed node, each recovery node came back to its previous original location. The stability assurance in connectivity and coverage is the main feature of the proposed algorithm. This assurance enhanced the overall lifetime of the network. The proposed method is a fusion of both localized and distributed algorithms. The overall messaging overhead for the proposed technique is minor for small networks. The success of the proposed scheme is proven by various simulations.

\section{Data Availability}

No data were used to support this study. We have conducted simulations to evaluate the performance of the proposed protocol. However, any query about the research conducted in this paper is highly appreciated and can be asked from the principal authors (Rab Nawaz Jadoon and Adnan Anwar Awan) upon request.

\section{Conflicts of Interest}

The authors declare no conflict of interest.

\section{Acknowledgments}

I, Dr. Rab Nawaz Jadoon, personally thank COMSATS University Islamabad-Abbottabad Campus and Key Laboratory of Wireless-Optical Communication, USTC Hefei, China, that extended their full support by providing us all key resources during the implementation of this project. I would also like to give special thanks to Prof. WuYang Zhou because of his continuous encouragement and research guidance throughout this work as a Head (Research Lab). This work is financially supported by the National Natural Science Foundation of China (Grant No. 61631018).

\section{References}

[1] M. Younis, S. Lee, S. Gupta, and K. Fisher, "A localized selfhealing algorithm for networks of moveable sensor nodes," in IEEE GLOBECOM 2008 - 2008 IEEE Global Telecommunications Conference, New Orleans, LA, USA, November 2008.

[2] K. Akkaya and M. Younis, "C2AP: coverage-aware and connectivity-constrained actor positioning in wireless sensor and actor networks," in 2007 IEEE International Performance, Computing, and Communications Conference, pp. 281-288, New Orleans, LA, USA, April 2007.

[3] M. Imran, M. Younis, A. Md Said, and H. Hasbullah, "Volunteer-instigated connectivity restoration algorithm for wireless sensor and actor networks," in 2010 IEEE International Con- ference on Wireless Communications, Networking and Information Security, pp. 679-683, Beijing, China, June 2010.

[4] Y. K. Joshi and M. Younis, "Restoring connectivity in a resource constrained WSN," Journal of Network and Computer Applications, vol. 66, pp. 151-165, 2016.

[5] P. Rawat, K. D. Singh, H. Chaouchi, and J. M. Bonnin, "Wireless sensor networks: a survey on recent developments and potential synergies," The Journal of Supercomputing, vol. 68, no. 1, pp. 1-48, 2014.

[6] Y. Zeng, C. J. Sreenan, N. Xiong, L. T. Yang, and J. H. Park, "Connectivity and coverage maintenance in wireless sensor networks," The Journal of Supercomputing, vol. 52, no. 1, pp. 23-46, 2010.

[7] A. K. Sangaiah, D. V. Medhane, T. Han, M. S. Hossain, and G. Muhammad, "Enforcing position-based confidentiality with machine learning paradigm through mobile edge computing in real-time industrial informatics," IEEE Transactions on Industrial Informatics, vol. 15, no. 7, pp. 4189-4196, 2019.

[8] A. K. Sangaiah, D. V. Medhane, G.-B. Bian, A. Ghoneim, M. Alrashoud, and M. S. Hossain, "Energy-aware green adversary model for cyberphysical security in the industrial system," IEEE Transactions on Industrial Informatics, vol. 16, no. 5, pp. 3322-3329, 2019.

[9] A. K. Sangaiah, M. Sadeghilalimi, A. A. R. Hosseinabadi, and W. Zhang, "Energy consumption in point-coverage wireless sensor networks via bat algorithm," IEEE Access, vol. 7, pp. 180258-180269, 2019.

[10] K. Akkaya, A. Thimmapuram, F. Senel, and S. Uludag, "Distributed recovery of actor failures in wireless sensor and actor networks," in 2008 IEEE Wireless Communications and Networking Conference, pp. 2480-2485, Las Vegas, NV, USA, March 2008.

[11] N. Tamboli and M. Younis, "Coverage-aware connectivity restoration in mobile sensor networks," Journal of Network and Computer Applications, vol. 33, no. 4, pp. 363-374, 2010.

[12] S. Chouikhi, I. El Korbi, Y. Ghamri-Doudane, and L. A. Saidane, "Fault-tolerant multi-channel allocation scheme for wireless sensor networks," in 2014 IEEE Wireless Communications and Networking Conference (WCNC), pp. 2438-2443, Istanbul, Turkey, April 2014.

[13] K. I. Gandhi, P. Narayanasamy, and P. Ushapreethi, "Coverage holes reduction in movable sensor networks using minimum movement technique," in 2011 International Conference on Recent Trends in Information Technology (ICRTIT), pp. 275280, Chennai, Tamil Nadu, India, June 2011.

[14] X. Wang, L. Xu, and S. Zhou, "Restoration strategy based on optimal relay node placement in wireless sensor networks," International Journal of Distributed Sensor Networks, vol. 11, no. 7, Article ID 409085, 2015.

[15] A. A. Abbasi, M. F. Younis, and U. A. Baroudi, "Recovering from a node failure in wireless sensor-actor networks with minimal topology changes," IEEE Transactions on Vehicular Technology, vol. 62, no. 1, pp. 256-271, 2012.

[16] A. Wichmann, T. Korkmaz, and A. S. Tosun, "Robot control strategies for task allocation with connectivity constraints in wireless sensor and robot networks," IEEE Transactions on Mobile Computing, vol. 17, no. 6, pp. 1429-1441, 2017.

[17] S. Shriwastav and D. Ghose, "Connectivity restoration in partitioned wireless sensor networks based on coverage importance using round table decision," in 2017 14th IEEE India 
Council International Conference (INDICON), pp. 1-6, Roorkee, India, December 2017.

[18] H. Mostafaei, M. U. Chowdhury, R. Islam, and H. Gholizadeh, "Connected P-Percent coverage in wireless sensor networks based on degree constraint dominating set approach," in Proceedings of the 18th ACM International Conference on Modeling, Analysis and Simulation of Wireless and Mobile Systems - MSWiM '15, pp. 157-160, New York, NY, USA, November 2015.

[19] H. Mostafaei, A. Montieri, V. Persico, and A. Pescapé, “An efficient partial coverage algorithm for wireless sensor networks," in 2016 IEEE Symposium on Computers and Communication (ISCC), pp. 501-506, New York, NY, USA, June 2016.

[20] H. Mostafaei and M. S. Obaidat, "Learning automaton-based self-protection algorithm for wireless sensor networks," IET Networks, vol. 7, no. 5, pp. 353-361, 2018.

[21] X. Liu, "Survivability-aware connectivity restoration for partitioned wireless sensor networks," IEEE Communications Letters, vol. 21, no. 11, pp. 2444-2447, 2017.

[22] O. Dagdeviren, V. K. Akram, and B. Tavli, "Design and evaluation of algorithms for energy-efficient and complete determination of critical nodes for wireless sensor network reliability," IEEE Transactions on Reliability, vol. 68, no. 1, pp. 280-290, 2018.

[23] P. Chanak, I. Banerjee, and R. S. Sherratt, "Energy-aware distributed routing algorithm to tolerate network failure in wireless sensor networks," Ad Hoc Networks, vol. 56, pp. 158-172, 2017. 